TRANSACTIONS OF THE

AMERICAN MATHEMATICAL SOCIETY

Volume 357 , Number 12, Pages 5097-5113

S 0002-9947(05)03702-5

Article electronically published on April 13, 2005

\title{
THE CYCLIC AND SIMPLICIAL COHOMOLOGY OF $l^{1}(\mathbf{N})$
}

\author{
FRÉDÉRIC GOURDEAU, B. E. JOHNSON, AND MICHAEL C. WHITE
}

\begin{abstract}
Let $\mathcal{A}=l^{1}\left(\mathbf{Z}_{+}\right)$be the unital semigroup algebra of $\mathbf{N}$. We show that the cyclic cohomology groups $\mathcal{H} C^{n}\left(\mathcal{A}, \mathcal{A}^{\prime}\right)$ vanish when $n$ is odd and are one dimensional when $n$ is even $(n \geq 2)$. Using Connes' exact sequence, these results are used to show that the simplicial cohomology groups $\mathcal{H}^{n}\left(\mathcal{A}, \mathcal{A}^{\prime}\right)$ vanish for $n \geq 2$. The results obtained are extended to unital algebras $l^{1}(S)$ for some other semigroups of $\mathbf{R}$.
\end{abstract}

\section{BACKGROUND AND DEFINITIONS}

In this paper, we continue the work done in GW, where it was shown that the third simplicial and cyclic cohomology groups of $l^{1}\left(\mathbf{Z}_{+}\right)$vanish.

Let us recall here that while $l^{1}\left(\mathbf{Z}_{+}\right)$, the unital semigroup algebra of $\mathbf{N}$, is not weakly amenable (see the proof of [BCD, Theorem 2.3]), it was shown that $\mathcal{H}^{2}\left(l^{1}\left(\mathbf{Z}_{+}\right), l^{1}\left(\mathbf{Z}_{+}\right)^{\prime}\right)$ [DaDu, Theorem 3.2] and $\mathcal{H}^{3}\left(l^{1}\left(\mathbf{Z}_{+}\right), l^{1}\left(\mathbf{Z}_{+}\right)^{\prime}\right)$ GW] are zero. These results suggested the conjecture that all of the simplicial cohomology groups of $l^{1}\left(\mathbf{Z}_{+}\right)$vanish for $n \geq 2$. In this paper, we show that this is indeed the case. We prove this through explicit calculations for the cyclic case, showing that all cyclic cohomology groups of odd degrees vanish and that all those of even degrees are one dimensional. We then deduce the result for simplicial cohomology groups of order two and more, using Connes' exact sequence.

We now recall some basic results and introduce our notation. Let $\mathcal{A}$ be a Banach algebra and let $\mathcal{A}^{\prime}$, the dual of $\mathcal{A}$, be a Banach $\mathcal{A}$-bimodule in the usual way. An $n$-cochain is a bounded $n$-linear map $T$ from $\mathcal{A}^{n}$ to $\mathcal{A}^{\prime}$ which we denote by $T \in C^{n}\left(\mathcal{A}, \mathcal{A}^{\prime}\right)$. The map $\delta^{n}: C^{n}\left(\mathcal{A}, \mathcal{A}^{\prime}\right) \rightarrow C^{n+1}\left(\mathcal{A}, \mathcal{A}^{\prime}\right)$ is defined by

$$
\begin{aligned}
\left(\delta^{n} T\right)\left(a_{1}, \ldots, a_{n+1}\right)\left(a_{0}\right)= & T\left(a_{2}, a_{3}, \ldots, a_{n+1}\right)\left(a_{0} a_{1}\right) \\
& +\sum_{j=1}^{n}(-1)^{j} T\left(a_{1}, a_{2}, \ldots, a_{j} a_{j+1}, \ldots, a_{n+1}\right)\left(a_{0}\right) \\
& +(-1)^{n+1} T\left(a_{1}, \ldots, a_{n}\right)\left(a_{n+1} a_{0}\right) .
\end{aligned}
$$

The $n$-cochain $T$ is an $n$-cocycle if $\delta^{n} T=0$ and it is an $n$-coboundary if $T=$ $\delta^{n-1} S$ for some $S \in C^{n-1}\left(\mathcal{A}, \mathcal{A}^{\prime}\right)$. The linear space of all $n$-cocycles is denoted by $\mathcal{Z}^{n}\left(\mathcal{A}, \mathcal{A}^{\prime}\right)$, and the linear space of all $n$-coboundaries is denoted by $\mathcal{B}^{n}\left(\mathcal{A}, \mathcal{A}^{\prime}\right)$. We also recall that $\mathcal{B}^{n}\left(\mathcal{A}, \mathcal{A}^{\prime}\right)$ is a subset of $\mathcal{Z}^{n}\left(\mathcal{A}, \mathcal{A}^{\prime}\right)$ and that the $n$ th-cohomology

Received by the editors November 14, 2002 and, in revised form, April 8, 2004.

2000 Mathematics Subject Classification. Primary 46H20, 46J40; Secondary 43A20, 16E40.

Key words and phrases. Cohomology, cyclic cohomology, simplicial cohomology, Banach algebra. 
group $\mathcal{H}^{n}\left(\mathcal{A}, \mathcal{A}^{\prime}\right)$ is defined by the quotient

$$
\mathcal{H}^{n}\left(\mathcal{A}, \mathcal{A}^{\prime}\right)=\frac{\mathcal{Z}^{n}\left(\mathcal{A}, \mathcal{A}^{\prime}\right)}{\mathcal{B}^{n}\left(\mathcal{A}, \mathcal{A}^{\prime}\right)}
$$

Note that in the above, by convention, $C^{0}\left(\mathcal{A}, \mathcal{A}^{\prime}\right)=\mathcal{A}^{\prime}$ and $C^{n}\left(\mathcal{A}, \mathcal{A}^{\prime}\right)=0$ for negative $n$.

The $n$-cochain $T$ is called cyclic if

$$
T\left(a_{1}, a_{2}, \ldots, a_{n}\right)\left(a_{0}\right)=(-1)^{n} T\left(a_{0}, a_{1}, \ldots, a_{n-1}\right)\left(a_{n}\right)
$$

and we denote the linear space of all cyclic $n$-cochains by $C C^{n}\left(\mathcal{A}, \mathcal{A}^{\prime}\right)$. It is well known (see [He1]) that the cyclic cochains $C C^{n}\left(\mathcal{A}, \mathcal{A}^{\prime}\right)$ form a subcomplex of $C^{n}\left(\mathcal{A}, \mathcal{A}^{\prime}\right)$, that is $\delta^{n}: C C^{n}\left(\mathcal{A}, \mathcal{A}^{\prime}\right) \rightarrow C C^{n+1}\left(\mathcal{A}, \mathcal{A}^{\prime}\right)$, and so we have cyclic versions of the spaces defined above which we denote by $\mathcal{B} C^{n}\left(\mathcal{A}, \mathcal{A}^{\prime}\right), \mathcal{Z} C^{n}\left(\mathcal{A}, \mathcal{A}^{\prime}\right)$ and $\mathcal{H} C^{n}\left(\mathcal{A}, \mathcal{A}^{\prime}\right)$. We will also use the notations $\mathcal{H} C^{n}(\mathcal{A})$ and $C C^{n}(\mathcal{A})$.

\section{The BASIC IDEA OF THE PROOF}

The proof proceeds by showing that the chain complex which defines the simplicial cohomology of $l^{1}\left(\mathbf{Z}_{+}\right)$is isomorphic to a chain complex which defines an equivariant simplicial cohomology, the basic idea being to work with the homology and cohomology defined on simplexes. Let us briefly explain this theory, as we will need an adaptation of it in our work.

Denote by $\Delta_{N}$ the $N$-simplex with vertices labelled $\{0,1, \ldots, N\}$. By a subsimplex (or face) of $\Delta_{N}$ we shall mean a list of vertices of $\Delta_{N}$ arranged in increasing order, allowing degenerate faces, that is, lists where the same vertex is repeated. Thus a face of dimension $n$ is an increasing list of $n+1$ numbers in the range $0, \ldots, N$.

Denote the space of $n$-chains on $\Delta_{N}$ by $C_{n}\left(\Delta_{N}\right)$, that is, the vector space with basis elements consisting of the $n$-dimensional faces of $\Delta_{N}$. The dual of $C_{n}\left(\Delta_{N}\right)$, which can be viewed as the space of complex valued functions on the $n$-dimensional faces, is denoted $C^{n}\left(\Delta_{N}\right)$. It is the space of $n$-cochains.

There is a boundary map $d^{n}: C_{n}\left(\Delta_{N}\right) \rightarrow C_{n-1}\left(\Delta_{N}\right)$ defined by

$$
d^{n}\left(v_{0}, v_{1}, \ldots, v_{n}\right)=\sum_{j=0}^{n}(-1)^{j}\left(v_{0}, v_{1}, \ldots, \hat{v}_{j}, \ldots, v_{n}\right),
$$

where $\hat{v}_{j}$ means that this entry is omitted. This induces a coboundary map $\delta^{n-1}$ : $C^{n-1}\left(\Delta_{N}\right) \rightarrow C^{n}\left(\Delta_{N}\right)$, which is simply the dual of $d^{n}$. We endow $C_{n}\left(\Delta_{N}\right)$ with the $l^{1}$-norm and its dual $C^{n}\left(\Delta_{N}\right)$ with the dual $l^{\infty}$-norm.

Remark 2.1. We will generally drop the superscripts for $d^{n}$ and $\delta^{n}$, and write $d$ and $\delta$.

The cohomology of these complexes gives us the simplicial homology and cohomology of the simplicial complex $\Delta_{N}$. It should be noted that the boundary operator sends increasing lists to increasing lists and thus sends faces to faces under our definition.

Normally one shows that the cohomology of $\Delta_{N}$ is trivial by using the fact that it is a cone. To do so, one constructs a map

$$
s: C_{n}\left(\Delta_{N}\right) \rightarrow C_{n+1}\left(\Delta_{N}\right)
$$


defined by $s\left(v_{0}, v_{1}, \ldots, v_{n}\right)=\left(0, v_{0}, \ldots, v_{n}\right)$. It is then easy to check that

$$
\begin{aligned}
d\left(s\left(v_{0}, v_{1}, \ldots, v_{n}\right)\right) & =d\left(0, v_{0}, \ldots, v_{n}\right) \\
& =\left(v_{0}, v_{1}, \ldots, v_{n}\right)-s\left(d\left(v_{0}, v_{1}, \ldots, v_{n}\right)\right) .
\end{aligned}
$$

Denoting the dual of this map by $s$ also, we have $\delta s R+s \delta R=R$ for every cochain $R \in C^{n}\left(\Delta_{N}\right)$. In particular, every cocycle is a coboundary. However, we are interested in calculating an equivariant cohomology, as we shall see, and we will need a different argument.

\section{TRANSFERRING THE PROBLEM}

From now on, $\mathcal{A}=l^{1}\left(\mathbf{Z}_{+}\right)$, where

$$
l^{1}\left(\mathbf{Z}_{+}\right)=\left\{f=\sum_{n=0}^{\infty} a_{n} z^{n}: \sum_{n=0}^{\infty} \bmod a_{n}<\infty\right\}
$$

with norm $\|f\|=\sum_{n=0}^{\infty} \bmod a_{n}$ and multiplication is given by the usual convolution multiplication on $\mathbf{Z}_{+}$.

An $n$-cochain $T \in C^{n}\left(\mathcal{A}, \mathcal{A}^{\prime}\right)$ is determined by its values

$$
T\left(z^{m_{1}}, z^{m_{2}}, \ldots, z^{m_{n}}\right)\left(z^{m_{0}}\right)
$$

where $m_{i} \in \mathbf{Z}_{+}, i=0, \ldots, n$. Let us note that, for $S \in C^{n-1}\left(\mathcal{A}, \mathcal{A}^{\prime}\right)$, to prove $\delta^{n-1} S=T$ it suffices to have

$$
\left(\delta^{n-1} S\right)\left(z^{m_{1}}, z^{m_{2}}, \ldots, z^{m_{n}}\right)\left(z^{m_{0}}\right)=T\left(z^{m_{1}}, z^{m_{2}}, \ldots, z^{m_{n}}\right)\left(z^{m_{0}}\right)
$$

for all choices of $m_{i} \in \mathbf{Z}_{+}, i=0, \ldots, n$.

Instead of working on simplexes as we introduced them in the previous section, it proves easier to work with integer $n$-tuples where translation is easily defined and is easy to work with. This is done as follows.

Given $T \in C^{n}\left(\mathcal{A}, \mathcal{A}^{\prime}\right)$ and $N \in \mathbf{Z}_{+}$, define the function $p_{N}(T)$ on integer $(n+1)$ tuples $\left(v_{0}, v_{1}, \ldots, v_{n}\right)$ such that $0 \leq v_{0} \leq v_{1} \leq \cdots \leq v_{n}$ and $v_{n}-v_{0} \leq N$ by

$$
p_{N}(T)\left(v_{0}, v_{1}, \ldots, v_{n}\right)=T\left(z^{-v_{0}+v_{1}}, z^{-v_{1}+v_{2}}, \ldots, z^{-v_{n-1}+v_{n}}\right)\left(z^{-v_{n}+N+v_{0}}\right) .
$$

Let $\Sigma_{N}$ denote the space of $(n+1)$-tuples $\left(v_{0}, v_{1}, \ldots, v_{n}\right)$ such that $0 \leq v_{0} \leq$ $v_{1} \leq \cdots \leq v_{n}$ and $v_{n}-v_{0} \leq N$, where $n$ is considered fixed for the moment. The function $p_{N}(T)$ which has just been defined does not act on the simplexes $\Delta_{N}$ introduced in the previous section but rather on $\Sigma_{N}$.

It is easily verified that the theory presented for the simplexes $\Delta_{N}$ also holds for $\Sigma_{N}$ and thus we can say that, in this sense, $p_{N}(T)$ is a cochain in $C^{n}\left(\Sigma_{N}\right)$. To avoid additional notation, we will also denote by $d$ and by $\delta$ the boundary and coboundary maps for $C_{n}\left(\Sigma_{N}\right)$ and $C^{n}\left(\Sigma_{N}\right)$, respectively.

As the next lemma shows, $\delta\left(p_{N} T\right)=p_{N}(\delta T)$, and thus we can transfer a problem on the complex $\left(C^{n}\left(\mathcal{A}, \mathcal{A}^{\prime}\right), \delta\right)$ to a series of problems on the complexes $\left(C^{n}\left(\Sigma_{N}\right), \delta\right)$.

Lemma 3.1. Let $T \in C^{n}\left(\mathcal{A}, \mathcal{A}^{\prime}\right)$ and fix $N \in \mathbf{Z}_{+}$. Then $\delta\left(p_{N} T\right)=p_{N}(\delta T)$.

Proof. Let $N$ be fixed and let $\mathbf{v}=\left(v_{0}, v_{1}, \ldots, v_{n}, v_{n+1}\right) \in C_{n+1}\left(\Sigma_{N}\right)$. Let $\mathbf{v}_{\hat{\jmath}}$ given by $\mathbf{v}_{\hat{\jmath}}=\left(v_{0}, v_{1}, \ldots, v_{j-1}, v_{j+1}, \ldots, v_{n+1}\right)$ denote $\mathbf{v}$ without the $j$ th component $(0 \leq j \leq n+1)$ so that $\delta\left(p_{N}(T)\right)(\mathbf{v})=\sum_{j=0}^{n+1}(-1)^{j} p_{N}(T)\left(\mathbf{v}_{\hat{\jmath}}\right)$

For $j=1, \ldots,(n+1), p_{N}(T)\left(\mathbf{v}_{\hat{\jmath}}\right)$ is given by

$$
T\left(z^{-v_{0}+v_{1}}, \ldots, z^{\left(-v_{j-1}+v_{j}\right)+\left(-v_{j}+v_{j+1}\right)}, \ldots, z^{-v_{n}+v_{n+1}}\right)\left(z^{-v_{n+1}+N+v_{0}}\right)
$$


which can be rewritten as

$$
T\left(z^{-v_{0}+v_{1}}, \ldots, z^{\left(-v_{j-1}+v_{j}\right)} z^{\left(-v_{j}+v_{j+1}\right)}, \ldots, z^{-v_{n}+v_{n+1}}\right)\left(z^{-v_{n+1}+N+v_{0}}\right) .
$$

For $j=0$, we have

$$
T\left(z^{-v_{1}+v_{2}}, z^{-v_{2}+v_{3}}, \ldots, z^{-v_{n-1}+v_{n}}\right)\left(z^{-v_{n}+N+v_{0}+\left(-v_{0}+v_{1}\right)}\right)
$$

which is equal to

$$
T\left(z^{-v_{1}+v_{2}}, z^{-v_{2}+v_{3}}, \ldots, z^{-v_{n-1}+v_{n}}\right)\left(z^{-v_{n}+N+v_{0}} z^{-v_{0}+v_{1}}\right) .
$$

Thus

$$
\begin{aligned}
& \sum_{j=0}^{n+1}(-1)^{j} p_{N}(T)\left(\mathbf{v}_{\hat{\jmath}}\right) \\
& \quad=\left(\delta^{n} T\right)\left(z^{-v_{0}+v_{1}}, z^{-v_{1}+v_{2}}, \ldots, z^{-v_{n}+v_{n+1}}\right)\left(z^{-v_{n+1}+N+v_{0}}\right) \\
& \quad=p_{N}\left(\delta^{n} T\right)\left(v_{0}, v_{1}, \ldots, v_{n+1}\right) .
\end{aligned}
$$

Thus $T \mapsto\left\{p_{N}(T)\right\}_{N=0}^{\infty}$ is a map of cochains, i.e. $T \mapsto\left\{p_{N}(T)\right\}_{N=0}^{\infty}$ commutes with the coboundary maps.

Definition 3.2. Let $T \in C^{n}\left(\Sigma_{N}\right)$.

(1) $T$ is cyclic if $T\left(v_{0}, v_{1}, \ldots, v_{n}\right)=(-1)^{n} T\left(v_{1}, v_{2}, \ldots, v_{n}, v_{0}+N\right)$ for all $\left(v_{0}, v_{1}, \ldots, v_{n}\right) \in C_{n}\left(\Sigma_{N}\right)$.

(2) $T$ is equivariant if it is invariant under translation, that is, if

$$
T\left(v_{0}, v_{1}, \ldots, v_{n}\right)=T\left(v_{0}+1, v_{1}+1, \ldots, v_{n}+1\right)
$$

for all $\left(v_{0}, v_{1}, \ldots, v_{n}\right) \in C_{n}\left(\Sigma_{N}\right)$.

It is easily verified that $T \in C^{n}\left(\mathcal{A}, \mathcal{A}^{\prime}\right)$ is cyclic if and only if $p_{N}(T)$ is cyclic for each $N$. It is also straightforward to show that the coboundary map $\delta$ sends cyclic cochains $T \in C^{n}\left(\Sigma_{N}\right)$ to cyclic ones. Thus cyclic cochains form a subcomplex and we will use the notation $T \in C C^{n}\left(\Sigma_{N}\right)$.

The definition of equivariant is a natural consequence of the definition of the map $T \mapsto p_{N}(T)$, for it is clear that $p_{N}(T)$ as defined is invariant under translation.

Let us now define an inverse map to $p_{N}$. Given a uniformly bounded family of cochains $\left\{T_{N}\right\}_{N=0}^{\infty} \in C^{n}\left(\Sigma_{N}\right)$, we define $\tilde{T} \in C^{n}\left(\mathcal{A}, \mathcal{A}^{\prime}\right)$ by

$$
\tilde{T}\left(z^{a_{1}}, z^{a_{2}}, \ldots, z^{a_{n}}\right)\left(z^{a_{0}}\right)=T_{N}\left(0, a_{1}, a_{1}+a_{2}, \ldots, \sum_{k=1}^{n} a_{k}\right),
$$

where $N=\sum_{k=0}^{n} a_{k}$. Note that because of the uniform bound on $\left\{T_{N}\right\}_{N \in \mathbf{Z}_{+}}$, it is clear that $\tilde{T}$ is bounded (and so is in $C^{n}\left(\mathcal{A}, \mathcal{A}^{\prime}\right)$ ).

It is easy to verify that if $\left\{p_{N}(T)\right\}_{N=0}^{\infty} \mapsto \tilde{T}$, then $\tilde{T}=T$. The converse is expressed as follows.

Lemma 3.3. If $\left\{T_{N}\right\}_{N \in \mathbf{Z}_{+}}$is a uniformly bounded family of equivariant cyclic cochains $T_{N} \in C C^{n}\left(\Sigma_{N}\right)$, then $\tilde{T}$ as defined above is cyclic and $p_{N}(\tilde{T})=T_{N}$. 
Proof. Let $\left\{T_{N}\right\}_{N=0}^{\infty}$ be a bounded family of equivariant cyclic cochains. Using both the equivariance and the cyclicity of $T_{N}$, and remembering that $N=a_{0}+$ $a_{1}+\cdots+a_{n}$, we have

$$
\begin{aligned}
\tilde{T} & \left(z^{a_{1}}, z^{a_{2}}, \ldots, z^{a_{n}}\right)\left(z^{a_{0}}\right) \\
& =T_{N}\left(0, a_{1}, a_{1}+a_{2}, \ldots, \sum_{k=1}^{n} a_{k}\right) \\
& =(-1)^{n} T_{N}\left(a_{1}, a_{1}+a_{2}, \ldots, \sum_{k=1}^{n} a_{k}, N\right) \quad \text { as } T_{N} \text { is cyclic } \\
& =(-1)^{n} T_{N}\left(0, a_{2}, \ldots, \sum_{k=2}^{n} a_{k}, N-a_{1}\right) \quad \text { as } T_{N} \text { is equivariant } \\
& =(-1)^{n} \tilde{T}\left(z^{a_{2}}, z^{a_{3}}, \ldots, z^{a_{n}}, z^{a_{0}}\right)\left(z^{a_{1}}\right) .
\end{aligned}
$$

Thus $\tilde{T}$ is cyclic.

To show that $p_{N}(\tilde{T})=T_{N}$, we only need to use equivariance as we have, for a given $N$,

$$
\begin{aligned}
p_{N}(\tilde{T})\left(v_{0}, \ldots v_{n}\right) & =\tilde{T}\left(z^{-v_{0}+v_{1}}, z^{-v_{1}+v_{2}}, \ldots, z^{-v_{n-1}+v_{n}}\right)\left(z^{-v_{n}+N+v_{0}}\right) \\
& =T_{N}\left(0,-v_{0}+v_{1},-v_{0}+v_{2}, \ldots,-v_{0}+v_{n}\right) \\
& =T_{N}\left(v_{0}, v_{1}, \ldots, v_{n}\right) .
\end{aligned}
$$

This lemma shows how the transfer will operate in the cyclic case, as we will be able to pull back to $C C^{n}\left(\mathcal{A}, \mathcal{A}^{\prime}\right)$ solutions found for $C C^{n}\left(\Sigma_{N}\right), N \in \mathbf{Z}_{+}$.

\section{THE CYCLIC CASE}

For a given $N>0$ and a given $n \geq 0$, let the maps $s_{m}: C_{n}\left(\Sigma_{N}\right) \rightarrow C_{n+1}\left(\Sigma_{N}\right)$, $m=0, \ldots, N-1$, be defined by

$$
s_{m}\left(v_{0}, v_{1}, \ldots, v_{n}\right)=(-1)^{j}\left(v_{0}, v_{1}, \ldots, v_{j-1}, \tilde{m}, v_{j}, \ldots, v_{n}\right),
$$

where $\tilde{m}$ and $j$ are such that $n-1 \geq j-1 \geq 0, \tilde{m} \cong m \bmod N$ and $v_{j-1}<\tilde{m} \leq v_{j}$. When no such $\tilde{m}$ and $j$ exist (i.e. when there is no integer congruent to $m \bmod N$ greater than $v_{0}$ and smaller than or equal to $v_{n}$ ), we define

$$
s_{m}\left(v_{0}, v_{1}, \ldots, v_{n}\right)=(-1)^{n+1}\left(v_{0}, v_{1}, \ldots, v_{j-1}, v_{j}, \ldots, v_{n}, \tilde{m}\right),
$$

where $\tilde{m}$ is the smallest integer congruent to $m \bmod N$ which is larger than $v_{n}$. Note that for the case $n=0$, the insertion is always after $v_{0}$.

For $N=0$, let the map $s_{0}: C_{n}\left(\Sigma_{0}\right) \rightarrow C_{n+1}\left(\Sigma_{0}\right)$ be defined by

$$
s_{0}(v, v, \ldots, v)=(v, v, \ldots, v) .
$$

Informally speaking, we insert a representative of the congruency class modulo $N$ at the first possible position between $v_{0}$ and $v_{n}$, inserting the appropriate representative after $v_{n}$ if no other position is available.

Let us denote by $\psi_{m}$ the dual of the map $s_{m}$. Thus $\psi_{m}: C^{n+1}\left(\Sigma_{N}\right) \rightarrow C^{n}\left(\Sigma_{N}\right)$.

Remark 4.1. Both $s_{m}$ and $\psi_{m}$ depend on $n$ and on $N$ and thus, to be correct, we should write $s_{m}^{n, N}$ and $\psi_{m}^{n, N}$ for these maps. However we drop the superscripts, as 
was done earlier. Thus, as we do in the next proposition, we may write $s_{m}$ as a map from $C_{n-1}\left(\Sigma_{N}\right)$ to $C_{n}\left(\Sigma_{N}\right)$ and $\psi_{m}$ as a map from $C^{n}\left(\Sigma_{N}\right)$ to $C^{n-1}\left(\Sigma_{N}\right)$.

Proposition 4.2. Let $N \in \mathbf{Z}_{+}$and let $T \in C C^{n}\left(\Sigma_{N}\right)$. Then, for $m=0,1, \ldots$, $N-1,\left(\delta \psi_{m}+\psi_{m} \delta\right) T=T$.

Proof. For $N=0$, we only have constant vectors $\mathbf{v}=(v, \ldots, v) \in C_{n}\left(\Sigma_{0}\right)$. Thus, if $n$ is even, $d \mathbf{v}=0$ and it is easily seen that $\left(d s_{0}+s_{0} d\right) \mathbf{v}=\mathbf{v}$. Similarly, if $n$ is odd, $\left(d s_{0}\right) \mathbf{v}=0$ and thus $\left(d s_{0}+s_{0} d\right) \mathbf{v}=\mathbf{v}$ easily follows. Dualizing, we have the result in the case $N=0$.

From now on, let $N>0$. For any $\mathbf{u}=\left(u_{0}, u_{1}, \ldots, u_{n}\right) \in C_{n}\left(\Sigma_{N}\right)$, let $\mathbf{u}_{\hat{\jmath}} \in$ $C_{n-1}\left(\Sigma_{N}\right), j=0, \ldots, n$, denote $\mathbf{u}$ without the $j$ th component of $\mathbf{u}$. With this notation, $d \mathbf{u}=\sum_{j=0}^{n}(-1)^{j} \mathbf{u}_{\hat{\jmath}}$. (Note that the proof is valid for the case $n=0$.)

Let $m$ and $\mathbf{v}=\left(v_{0}, v_{1}, \ldots, v_{n}\right)$ be fixed, $\mathbf{v} \in C_{n}\left(\Sigma_{N}\right)$, and let

$$
s_{m}(\mathbf{v})=(-1)^{k}\left(v_{0}, v_{1}, \ldots, v_{k-1}, \tilde{m}, v_{k}, v_{k+1}, \ldots, v_{n}\right) .
$$

Let $\mathbf{w}$ be defined by

$$
\mathbf{w}=(-1)^{k} s_{m}(\mathbf{v})=\left(v_{0}, v_{1}, \ldots, v_{k-1}, \tilde{m}, v_{k}, v_{k+1}, \ldots, v_{n}\right),
$$

where $1 \leq k \leq n+1$. Note that $k=n+1$ corresponds to $s_{m}(\mathbf{v})=(-1)^{n+1} \mathbf{w}=$ $(-1)^{n+1}\left(v_{0}, v_{1}, \ldots, v_{n}, \tilde{m}\right)$. Thus, from now on, $k$ and $\tilde{m}$ are also fixed.

We want to consider the value of $\left(d s_{m}+s_{m} d\right)(\mathbf{v})$. For $d s_{m}(\mathbf{v})$, we get

$$
d s_{m}(\mathbf{v})=(-1)^{k}\left[\sum_{j=0}^{k-1}(-1)^{j} \mathbf{w}_{\hat{\jmath}}+(-1)^{k} \mathbf{v}+\sum_{j=k}^{n}(-1)^{j+1} \mathbf{w}_{\widehat{j+1}}\right] .
$$

For $s_{m} d(\mathbf{v})$, we have

$$
s_{m} d(\mathbf{v})=s_{m}\left(\sum_{j=0}^{n}(-1)^{j} \mathbf{v}_{\hat{\jmath}}\right)=\sum_{j=0}^{n}(-1)^{j} s_{m}\left(\mathbf{v}_{\hat{\jmath}}\right) .
$$

Some care needs to be taken to evaluate this last sum properly. We have

$$
s_{m}\left(\mathbf{v}_{\hat{\jmath}}\right)= \begin{cases}(-1)^{k-1} \mathbf{w}_{\hat{\jmath}} & \text { if } j \leq k-1 \text { and } k \neq 1 \\ (-1)^{k} \mathbf{w}_{\widehat{j+1}} & \text { if } j \geq k \\ (-1)^{n}\left(v_{1}, v_{2}, \ldots, v_{n}, \tilde{m}+N\right) & \text { if } j=0 \text { and } k=1 .\end{cases}
$$

Thus we get

$$
s_{m} d(\mathbf{v})= \begin{cases}(-1)^{k-1} \sum_{j=0}^{k-1}(-1)^{j} \mathbf{w}_{\hat{\jmath}}+(-1)^{k} \sum_{j=k}^{n}(-1)^{j} \mathbf{w}_{\widehat{j+1}} & \text { if } k>1 \\ (-1)^{n}\left(v_{1}, \ldots, v_{n}, \tilde{m}+N\right)+(-1) \sum_{j=1}^{n}(-1)^{j} \mathbf{w}_{\widehat{j+1}} & \text { if } k=1 .\end{cases}
$$

We can combine these expressions to obtain

$$
\left(d s_{m}+s_{m} d\right)(\mathbf{v})=\mathbf{v}
$$

if $k>1$, and

$$
\left(d s_{m}+s_{m} d\right)(\mathbf{v})=\mathbf{v}-\mathbf{w}_{\tilde{0}}+(-1)^{n}\left(v_{1}, v_{2}, \ldots, v_{n}, \tilde{m}+N\right)
$$

if $k=1$.

Noting that $\mathbf{w}_{\hat{0}}=\left(\tilde{m}, v_{1}, v_{2}, \ldots, v_{n}\right)$, to prove $\left(\delta \psi_{m}+\psi_{m} \delta\right) T=T$ we now only need to show that

$$
-T\left(\tilde{m}, v_{1}, v_{2}, \ldots, v_{n}\right)+(-1)^{n} T\left(v_{1}, v_{2}, \ldots, v_{n}, \tilde{m}+N\right)=0 .
$$


As $T$ is cyclic, we have

$$
T\left(\tilde{m}, v_{1}, v_{2}, \ldots, v_{n}\right)=(-1)^{n} T\left(v_{1}, v_{2}, \ldots, v_{n}, \tilde{m}+N\right),
$$

and the result follows immediately.

The last proposition shows that if $T \in C C^{n}\left(\Sigma_{N}\right)$ is a cocycle (that is, if $\delta T=0$ ), then $\delta\left(\psi_{m} T\right)=T$. Let $S=\psi_{m} T$. We know that $S \in C^{n-1}\left(\Sigma_{N}\right)$, however, we also need $S$ cyclic and equivariant to be able to pull back this solution to $l^{1}\left(\mathbf{Z}_{+}\right)$.

Some work will be needed to obtain an equivariant solution but, as the next proposition shows, $S$ defined as above is cyclic except possibly at one point, the degenerate vector $\mathbf{v}=(v, \ldots, v)$, when $N=0$.

Proposition 4.3. Let $T \in C C^{n}\left(\Sigma_{N}\right)$.

(1) If $N>0$, then $\psi_{m}(T)$ is cyclic for $m=0,1, \ldots, N-1$.

(2) If $N=0$ and $n$ is odd, then $\psi_{0}(T)$ is cyclic.

(3) If $N=0$ and $n$ is even, then $\psi_{0}(T)$ is cyclic iff $T(v, \ldots, v)=0$.

Proof. To prove (1), let $T \in C C^{n}\left(\Sigma_{N}\right)$, where $N>0$, so that $\psi_{m} T \in C^{n-1}\left(\Sigma_{N}\right)$. Let $\mathbf{v}=\left(v_{0}, v_{1}, \ldots, v_{n-1}\right) \in C_{n-1}\left(\Sigma_{N}\right)$. We wish to show that

$$
\psi_{m} T(\mathbf{v})=(-1)^{n-1} \psi_{m} T\left(v_{1}, v_{2}, \ldots, v_{n-1}, v_{0}+N\right) .
$$

Let $\mathbf{w}$ be defined as in the proof of the previous proposition, $\psi_{m} T(\mathbf{v})=$ $(-1)^{k} T(\mathbf{w})$. Then, if $k>1$, we have

$$
\begin{array}{rl}
(-1)^{n-1} \psi_{m} & T\left(v_{1}, v_{2}, \ldots, v_{n-1}, v_{0}+N\right) \\
& =(-1)^{n-1}(-1)^{k-1} T\left(v_{1}, \ldots, v_{k-1}, \tilde{m}, v_{k}, \ldots, v_{n-1}, v_{0}+N\right) \\
& =(-1)^{n+k-2}(-1)^{n} T\left(v_{0}, v_{1}, \ldots, v_{k-1}, \tilde{m}, v_{k}, \ldots, v_{n-1}\right) \\
& =(-1)^{k} T\left(v_{0}, v_{1}, \ldots, v_{k-1}, \tilde{m}, v_{k}, \ldots, v_{n-1}\right) \\
& =(-1)^{k} T(\mathbf{w})=\psi_{m} T(\mathbf{v}) .
\end{array}
$$

If $k=1$, we have

$$
\begin{array}{rl}
(-1)^{n-1} \psi_{m} & T\left(v_{1}, v_{2}, \ldots, v_{n-1}, v_{0}+N\right) \\
= & (-1)^{n-1}(-1)^{n} T\left(v_{1}, \ldots, v_{n-1}, v_{0}+N, \tilde{m}+N\right) \\
= & (-1)^{1}(-1)^{n} T\left(\tilde{m}, v_{1}, \ldots, v_{n-1}, v_{0}+N\right) \\
= & (-1)^{n-1}(-1)^{n} T\left(v_{0}, \tilde{m}, v_{1}, \ldots, v_{n-1}\right) \\
& =-T(\mathbf{w})=\psi_{m} T(\mathbf{v})
\end{array}
$$

which completes the proof of the first statement.

When $N=0$, then $\psi_{0} T$ is cyclic if $\psi_{0} T(v, \ldots, v)=(-1)^{n-1} \psi_{0} T(v, \ldots, v)$. This is no requirement if $n$ is odd (and, furthermore, $T$ being cyclic implies that $T(v, \ldots, v)=0$ for $n$ odd $)$. When $n$ is even, it is true if and only if $T(v, \ldots, v)=0$, and the proof is completed.

To obtain an equivariant solution, we will take an average. For $N=0$, we let $\bar{\psi}=\psi_{0}$ and $\bar{s}=s_{0}$. For $N>0$, we let $\bar{\psi}$ and $\bar{s}$ be defined by

$$
\bar{\psi}=\frac{1}{N} \sum_{m=0}^{N-1} \psi_{m} \quad \text { and } \quad \bar{s}=\frac{1}{N} \sum_{m=0}^{N-1} s_{m} .
$$

Then $S=\bar{\psi} T$ is the equivariant and cyclic cochain we were looking for. 
Remark that for $N=0$, if $T \in C C^{n}\left(\Sigma_{0}\right)$ is equivariant, then so is $S=\bar{\psi} T$ (with $\left.S \in C C^{n-1}\left(\Sigma_{0}\right)\right)$. It then follows from Proposition 4.2 that if $T$ is a cocycle, then $\delta S=T$. Thus, for $N=0$, we get an equivariant $S$ such that $\delta S=T$, which will furthermore be cyclic if $n$ is odd or if $T(v, \ldots, v)=0$.

For $N \neq 0$, we have the following theorem.

Theorem 4.4. Let $N>0$ and let $T \in C C^{n}\left(\Sigma_{N}\right)$ be an equivariant cyclic cocycle. Then there exists $S \in C C^{n-1}\left(\Sigma_{N}\right)$, an equivariant cyclic cochain such that $\delta S=T$ and $\|S\| \leq\|T\|$.

Proof. Let $N>0$. We know from Proposition 4.3 that $S=\bar{\psi} T$ is cyclic, and it follows from Proposition 4.2 that $\delta S=T$ if $T$ is a cocycle. It is also straightforward that $\left\|\psi_{m} T\right\| \leq\|T\|$ for each $m$, and so $\|S\| \leq\|T\|$. Hence we only need to show that $S=\bar{\psi} T$ is equivariant.

Let $e \mathbf{v}=e\left(v_{0}, v_{1}, \ldots, v_{n}\right)=\left(v_{0}+1, v_{1}+1, \ldots, v_{n}+1\right)$. We want to show that $\bar{\psi} T(\mathbf{v})=\bar{\psi} T(e \mathbf{v})$. However $s_{0}(e \mathbf{v})=e\left(s_{N-1} \mathbf{v}\right)$ and $s_{k}(e \mathbf{v})=e\left(s_{k-1} \mathbf{v}\right)$ for $k=1,2, \ldots, N-1$. Thus $\bar{\psi} T(e \mathbf{v})=T(\bar{s}(e \mathbf{v}))=T(e(\bar{s} \mathbf{v}))=T(\bar{s} \mathbf{v})$, where the last equality holds as $T$ is equivariant. This proves the result as $T(\bar{s} \mathbf{v})=\bar{\psi} T(\mathbf{v})$ by definition.

In view of the statements to come, we adopt the following definition.

Definition 4.5. Let $T \in C C^{n}(\mathcal{A})$ where $n \geq 0$.

(1) $T$ is reduced cyclic if $T\left(z^{0}, z^{0}, \ldots, z^{0}\right)\left(z^{0}\right)=0$.

(2) The cohomology of reduced cyclic cochains will be called the reduced cyclic cohomology.

We now have the results in place to prove that the reduced cyclic cohomology of $l^{1}\left(\mathbf{Z}_{+}\right)$vanishes.

Theorem 4.6. $\mathcal{H} C^{n}\left(l^{1}\left(\mathbf{Z}_{+}\right)\right)$is trivial when $n$ is odd, and is one dimensional when $n$ is even, $n \geq 2$.

Proof. Recall that $\mathcal{A}=l^{1}\left(\mathbf{Z}_{+}\right)$. Given a cyclic $n$-cocycle $T \in C C^{n}(\mathcal{A})$, we consider $\left\{p_{N}(T)\right\}_{N=0}^{\infty}$. By Lemma 3.1 this is a sequence of equivariant cocycles in $C C^{n}\left(\Sigma_{N}\right)$ $\left(N \in \mathbf{Z}_{+}\right)$, which is clearly uniformly bounded by $\|T\|$.

When $n$ is odd, by Theorem 4.4 (and by the remark preceding it in the case $N=0$ ), we know that $\left\{p_{N}(T)\right\}_{N=0}^{\infty}$ is the coboundary of a uniformly bounded family $\left\{S_{N}\right\}_{N=0}^{\infty}$ of equivariant cochains in $C C^{n-1}\left(\Sigma_{N}\right)$. This family defines a cyclic cochain $\tilde{S}$, by Lemma 3.3. whose coboundary is $T$, by Lemma 3.1 and Lemma 3.3 . Thus $T$ is a coboundary and the cyclic cohomology vanishes.

When $n$ is even, the same is true if $T$ is reduced cyclic, again by Theorem 4.4 and by the remark preceding it in the case $N=0$.

To show that $\mathcal{H} C^{n}\left(l^{1}\left(\mathbf{Z}_{+}\right)\right)$is not trivial for $n$ even $(n \geq 2)$, let us consider the cochain $\tau \in C C^{n}(\mathcal{A})$ given by

$$
\tau\left(f_{1}, \ldots, f_{n}\right)\left(f_{0}\right)=\prod_{i=0}^{n} f_{i}(0),
$$

where $f_{i} \in \mathcal{A}, i=0, \ldots, n$. It is clear that $\tau$ is cyclic, as claimed, and it is also clear that $\delta \tau=0$, and thus $\tau$ is a cyclic $n$-cocycle. 
If $\tau$ cobounds, say $\tau=\delta S$, then $S$ would be a cyclic cochain in $C C^{n-1}(\mathcal{A})$ such that

$$
1=\tau\left(z^{0}, \ldots, z^{0}\right)\left(z^{0}\right)=\delta S\left(z^{0}, \ldots, z^{0}\right)\left(z^{0}\right) .
$$

As $n-1$ is odd and $S$ cyclic, we have $\delta S\left(z^{0}, \ldots, z^{0}\right)\left(z^{0}\right)=S\left(z^{0}, \ldots, z^{0}\right)\left(z^{0}\right)=0$. However $\tau\left(z^{0}, \ldots, z^{0}\right)\left(z^{0}\right)=1$ and thus $\tau$ does not cobound.

To show that $\mathcal{H} C^{n}\left(l^{1}\left(\mathbf{Z}_{+}\right)\right)$is one dimensional, consider an arbitrary cocycle $T \in C C^{n}(\mathcal{A})$ and let $\lambda=T\left(z^{0}, \ldots, z^{0}\right)\left(z^{0}\right)$. Then $T_{\lambda}=T-\lambda \tau$ is reduced cyclic and thus cobounds by the first part of the proof.

Corollary 4.7. The reduced cyclic cohomology groups of $l^{1}\left(\mathbf{Z}_{+}\right)$are trivial for all orders $n \geq 1$.

Proof. This follows immediately from the proof of the previous theorem.

It will be useful to consider the ideal $\mathcal{I}$ of $\mathcal{A}$ given by

$$
\mathcal{I}=\left\{f=\sum_{n=0}^{\infty} a_{n} z^{n} \in \mathcal{A}: a_{0}=0\right\} .
$$

Corollary 4.8. With the notation above, $\mathcal{H} C^{n}(\mathcal{I})=0$ for $n \geq 1$.

Proof. Any $T \in C C^{n}(\mathcal{I})$ can be extended to $T \in C C^{n}(\mathcal{A})$ by first defining

$$
T\left(z^{a_{0}}, \ldots, z^{a_{n-1}}\right)\left(z^{a_{n}}\right)=0
$$

if $a_{i}=0$ for some $i, 0 \leq i \leq n$, and then extending it by linearity. Following the proof of Theorem 4.6, we get a cyclic cochain $\tilde{S}$ whose coboundary is $T$. We can always restrict $\tilde{S} \in C C^{n-1}(\mathcal{A})$ to $\tilde{S} \in C C^{n-1}(\mathcal{I})$, and the coboundary of this restricted $\tilde{S}$ is the original $T \in C C^{n}(\mathcal{I})$. Thus the cyclic cohomology vanishes.

We can now prove that all simplicial cohomology groups of $\mathcal{A}=l^{1}\left(\mathbf{Z}_{+}\right)$of order at least two vanish.

Theorem 4.9. $\mathcal{H}^{n}\left(l^{1}\left(\mathbf{Z}_{+}\right), l^{1}\left(\mathbf{Z}_{+}\right)^{\prime}\right)$ is trivial for $n \geq 2$.

Proof. The part of Connes' exact sequence below exists and is exact GW, Lemma $2.2]$

$$
\begin{aligned}
& \mathcal{H} C^{2}\left(\mathcal{I}, \mathcal{I}^{\prime}\right) \rightarrow \mathcal{H}^{2}\left(\mathcal{I}, \mathcal{I}^{\prime}\right) \rightarrow \mathcal{H} C^{1}\left(\mathcal{I}, \mathcal{I}^{\prime}\right) \rightarrow \mathcal{H} C^{3}\left(\mathcal{I}, \mathcal{I}^{\prime}\right) \\
& \rightarrow \mathcal{H}^{3}\left(\mathcal{I}, \mathcal{I}^{\prime}\right) \rightarrow \mathcal{H} C^{2}\left(\mathcal{I}, \mathcal{I}^{\prime}\right) \rightarrow \mathcal{H} C^{4}\left(\mathcal{I}, \mathcal{I}^{\prime}\right) \rightarrow \mathcal{H}^{4}\left(\mathcal{I}, \mathcal{I}^{\prime}\right) \rightarrow \ldots
\end{aligned}
$$

and we immediately deduce that if all cyclic cohomology groups for $n \geq 1$ vanish, then all simplicial cohomology groups of order at least 2 vanish also. Thus $\mathcal{H}^{n}\left(\mathcal{I}, \mathcal{I}^{\prime}\right)=0$ for $n \geq 2$. As we know that for $n \geq 2, \mathcal{H}^{n}\left(\mathcal{A}, \mathcal{A}^{\prime}\right)$ is isomorphic to $\mathcal{H}^{n}\left(\mathcal{I}, \mathcal{I}^{\prime}\right)$ GW, Proposition 2.1], the proof is completed.

\section{An EXPLICIT FORMUla FOR THE CONTRACTING HOMOTOPY}

The map $\bar{\psi}$ which we have constructed can be pulled back to obtain the map $\sigma: C^{n+1}\left(\mathcal{A}, \mathcal{A}^{\prime}\right) \rightarrow C^{n}\left(\mathcal{A}, \mathcal{A}^{\prime}\right)$. This is given by

$$
\begin{aligned}
& \sigma T\left(z^{a_{0}}, z^{a_{1}}, \ldots, z^{a_{n-1}}\right)\left(z^{a_{n}}\right) \\
& \quad=\frac{1}{N} \sum_{k=0}^{n}(-1)^{k+1} \sum_{j=0}^{a_{k}-1} T\left(z^{a_{0}}, \ldots, z^{a_{k}-j}, z^{j}, z^{a_{k+1}}, \ldots, z^{a_{n-1}}\right)\left(z^{a_{n}}\right),
\end{aligned}
$$


where $N=\sum_{i=0}^{n} a_{i}>0$. Note that in the sum above, the case $k=n$ corresponds to $\frac{1}{N}(-1)^{n+1} \sum_{j=0}^{a_{n}-1} T\left(z^{a_{0}}, z^{a_{1}}, \ldots, z^{a_{n-1}}, z^{a_{n}-j}\right)\left(z^{j}\right)$, and also note that the sum is empty whenever some $a_{k}=0$.

Remark 5.1. Contrary to the notation introduced in the first section, we let $z^{a_{0}}$ denote the first component of the $n$-dimensional chain and not the element of the module $\mathcal{A}$. This change of notation enables us to write the map $\sigma$ in a more compact form and to simplify the notation used in the proof of Proposition 5.3 .

When all $a_{i}$ are zero, i.e. in the case $N=0$, we define

$$
\sigma T\left(z^{0}, z^{0}, \ldots, z^{0}\right)\left(z^{0}\right)=T\left(z^{0}, z^{0}, \ldots, z^{0}\right)\left(z^{0}\right),
$$

where the vectors have dimensions $n$ and $n+1$, respectively.

We need $\sigma: C C^{n+1}\left(\mathcal{A}, \mathcal{A}^{\prime}\right) \rightarrow C C^{n}\left(\mathcal{A}, \mathcal{A}^{\prime}\right)$, i.e. we need $\sigma$ to send cyclic cochains to cyclic ones. As we proved earlier, this will be the case in odd dimensions and in the case of reduced cyclic cochains.

Lemma 5.2. Let $T \in C C^{n+1}\left(\mathcal{A}, \mathcal{A}^{\prime}\right)$.

(1) If $n+1$ is odd, then $\sigma T$ is cyclic.

(2) If $n+1$ is even, then $\sigma T$ is cyclic if and only if $T$ is reduced.

Proof. The proof is straightforward. We want to determine when the following are equal:

$$
\sigma T\left(z^{a_{0}}, \ldots, z^{a_{n-1}}\right)\left(z^{a_{n}}\right) \quad \text { and } \quad(-1)^{n} \sigma T\left(z^{a_{1}}, \ldots, z^{a_{n}}\right)\left(z^{a_{0}}\right) .
$$

When $n+1$ is odd, $T$ cyclic implies $T$ reduced. Thus, for $n+1$ odd and $a_{0}=a_{1}=\cdots=a_{n}=0$, both expressions vanish and are equal.

When $n+1$ is even, $T$ cyclic does not imply that $T$ is reduced. Thus, when $a_{0}=a_{1}=\cdots=a_{n}=0$ and $n+1$ is even, the two expressions are of opposite signs unless they are both zero, which is the case if and only if $T$ is reduced.

We now show that when at least one $a_{i}$ is not zero, we always have

$$
\sigma T\left(z^{a_{0}}, \ldots, z^{a_{n-1}}\right)\left(z^{a_{n}}\right)=(-1)^{n} \sigma T\left(z^{a_{1}}, \ldots, z^{a_{n}}\right)\left(z^{a_{0}}\right),
$$

where we note that

$$
\begin{aligned}
& \sigma T\left(z^{a_{1}}, \ldots, z^{a_{n}}\right)\left(z^{a_{0}}\right) \\
& =\frac{1}{N}\left[\sum_{k=1}^{n}(-1)^{k} \sum_{j=0}^{a_{k}-1} T\left(z^{a_{1}}, \ldots, z^{a_{k}-j}, z^{j}, z^{a_{k+1}}, \ldots, z^{a_{n}}\right)\left(z^{a_{0}}\right)\right. \\
& \left.\quad+\quad(-1)^{n+1} \sum_{j=0}^{a_{0}-1} T\left(z^{a_{1}}, \ldots, z^{a_{n}}, z^{a_{0}-j}\right)\left(z^{j}\right)\right]
\end{aligned}
$$

Now

$$
\begin{aligned}
& \sigma T\left(z^{a_{0}}, \ldots, z^{a_{n-1}}\right)\left(z^{a_{n}}\right) \\
& \quad=\frac{1}{N} \sum_{k=0}^{n}(-1)^{k+1} \sum_{j=0}^{a_{k}-1} T\left(z^{a_{0}}, \ldots, z^{a_{k}-j}, z^{j}, z^{a_{k+1}}, \ldots, z^{a_{n-1}}\right)\left(z^{a_{n}}\right) .
\end{aligned}
$$


Using that $T$ is cyclic, we have, for $k \geq 1$,

$$
\begin{aligned}
& (-1)^{k+1} \sum_{j=0}^{a_{k}-1} T\left(z^{a_{0}}, \ldots, z^{a_{k}-j}, z^{j}, z^{a_{k+1}}, \ldots, z^{a_{n-1}}\right)\left(z^{a_{n}}\right) \\
& \quad=(-1)^{k+1}(-1)^{n+1} \sum_{j=0}^{a_{k}-1} T\left(z^{a_{1}}, \ldots, z^{a_{k}-j}, z^{j}, z^{a_{k+1}}, \ldots, z^{a_{n}}\right)\left(z^{a_{0}}\right)
\end{aligned}
$$

and, for $k=0$, cycling twice gives

$$
-\sum_{j=0}^{a_{0}-1} T\left(z^{a_{0}-j}, z^{j}, z^{a_{1}}, \ldots, z^{a_{n-1}}\right)\left(z^{a_{n}}\right)=-\sum_{j=0}^{a_{0}-1} T\left(z^{a_{1}}, \ldots, z^{a_{n}}, z^{a_{0}-j}\right)\left(z^{j}\right) .
$$

Combining these gives the result.

The work done so far permits us to state without proof the following proposition, insofar as we check that the map $\sigma$ corresponds to the map $\bar{\psi}$. However we will give a direct proof which will enable us to generalize our results.

Proposition 5.3. Let $T \in C C^{n}\left(\mathcal{A}, \mathcal{A}^{\prime}\right), n \geq 1$. Then

$$
(\sigma \delta+\delta \sigma) T=T .
$$

Proof. Let $T\left[a_{0}, a_{1}, \ldots, a_{n}\right]$ denote $T\left(z^{a_{0}}, \ldots, z^{a_{n-1}}\right)\left(z^{a_{n}}\right)$.

In the degerate case $a_{0}=a_{1}=\cdots=a_{n}=0$, the result is easily verified. (Recall however that in the case where $n$ is even, $\sigma T$ is cyclic if and only if $T$ is reduced cyclic.)

For the general case, let us first consider $\delta(\sigma T)\left[a_{0}, \ldots, a_{n}\right]$. This is given by $\sigma T\left[a_{1}, \ldots, a_{n-1}, a_{n}+a_{0}\right]+\sum_{l=1}^{n}(-1)^{l} \sigma T\left[a_{0}, \ldots, a_{l-1}+a_{l}, \ldots, a_{n}\right]$. Applying $\sigma$ directly therefore gives a triple summand which we divide as follows, leaving out the coefficient $1 / N$ everywhere.

When $l \neq 0$, we have

$$
\begin{aligned}
& \sum_{l=1}^{n} \sum_{k>l} \sum_{j=0}^{a_{k}-1}(-1)^{l}(-1)^{k} T\left[a_{0}, \ldots, a_{l-1}+a_{l}, \ldots, a_{k}-j, j, \ldots, a_{n}\right], \\
& \sum_{l=1}^{n} \sum_{j=0}^{a_{l-1}+a_{l}-1}(-1)^{l}(-1)^{l} T\left[a_{0}, \ldots, a_{l-1}+a_{l}-j, j, \ldots, a_{n}\right], \\
& \sum_{l=1}^{n} \sum_{k<l-1} \sum_{j=0}^{a_{k}-1}(-1)^{l}(-1)^{k+1} T\left[a_{0}, \ldots, a_{k}-j, j, \ldots, a_{l-1}+a_{l}, \ldots, a_{n}\right] .
\end{aligned}
$$

Here (2) corresponds to $k=l-1$. Also, there is no occurence of $k=l$ as the term $a_{l-1}+a_{l}$ excludes this value of $k$.

When $l=0$, we have

$$
\begin{gathered}
\sum_{k=1}^{n-1} \sum_{j=0}^{a_{k}-1}(-1)^{k} T\left[a_{1}, \ldots, a_{k}-j, j, \ldots, a_{n}+a_{0}\right], \\
\sum_{j=0}^{a_{n}+a_{0}-1}(-1)^{n} T\left[a_{1}, a_{2}, \ldots, a_{n}+a_{0}-j, j\right] .
\end{gathered}
$$

Note that there is no occurence of $k=0$ as $l=0$ excludes this possibility. 
In the expressions above, adding up two consecutive coordinates (which is the effect of $\delta$ ) occurs prior to the splitting of a term in two (which is the effect of $\sigma$ ) and thus all terms include $(-1)^{l}$, while they have either $(-1)^{k+1}$ or $(-1)^{k}$ depending on the relative values of $l$ and $k$.

When considering $\sigma \delta T$, the opposite occurs. All terms include $(-1)^{k+1}$, while they have either $(-1)^{l}$ or $(-1)^{l+1}$, depending again on the relative values of $l$ and $k$. This will cause the exact cancellation of most terms.

We express $\sigma \delta T$ as the sum of the following expressions, leaving out once again the coefficient $1 / N$. When $l \neq 0$, we have

$$
\begin{aligned}
& \sum_{l=1}^{n} \sum_{k>l} \sum_{j=0}^{a_{k}-1}(-1)^{k+1}(-1)^{l} T\left[a_{0}, \ldots, a_{l-1}+a_{l}, \ldots, a_{k}-j, j, \ldots, a_{n}\right], \\
& \sum_{k=1}^{n} \sum_{j=0}^{a_{k}-1}(-1)^{k+1}(-1)^{k} T\left[a_{0}, \ldots, a_{k-1}+a_{k}-j, j, \ldots, a_{n}\right], \\
& \sum_{k=0}^{n} \sum_{j=0}^{a_{k}-1}(-1)^{k+1}(-1)^{k+1} T\left[a_{0}, \ldots, a_{k}, \ldots, a_{n}\right] \\
& \sum_{k=0}^{n-1} \sum_{j=0}^{a_{k}-1}(-1)^{k+1}(-1)^{k+2} T\left[a_{0}, \ldots, a_{k}-j, j+a_{k+1}, \ldots, a_{n}\right] \\
& \sum_{l=1}^{n} \sum_{k<l-1} \sum_{j=0}^{a_{k}-1}(-1)^{k+1}(-1)^{l+1} T\left[a_{0}, \ldots, a_{k}-j, j, \ldots, a_{l-1}+a_{l}, \ldots, a_{n}\right] .
\end{aligned}
$$

Here note that (7), (8) and (9) correspond to cases expected for $k=l$ and $k=l-1$. There are three cases, as an extra one arises from the action of $\sigma$.

When $l=0$, we have

$$
\begin{aligned}
& \sum_{k=1}^{n-1} \sum_{j=0}^{a_{k}-1}(-1)^{k+1} T\left[a_{1}, \ldots, a_{k}-j, j, \ldots, a_{n}+a_{0}\right], \\
& \sum_{j=0}^{a_{0}-1}(-1) T\left[j, a_{1}, \ldots, a_{n-1}, a_{n}+a_{0}-j\right], \\
& \sum_{j=0}^{a_{n}-1}(-1)^{n+1} T\left[a_{1}, \ldots, a_{n-1}, a_{n}-j, j+a_{0}\right] .
\end{aligned}
$$

Considering the expressions above for $(\sigma \delta+\delta \sigma) T$, we see that (1), (3) and (4) cancel out exactly (6), (10) and (11).

Now (9) which is $-\sum_{k=0}^{n-1} \sum_{j=0}^{a_{k}-1} T\left[a_{0}, \ldots, a_{k}-j, j+a_{k+1}, \ldots, a_{n}\right]$ can be rewritten as $-\sum_{k=1}^{n} \sum_{j=0}^{a_{k-1}-1} T\left[a_{0}, \ldots, a_{k-1}-j, j+a_{k}, \ldots, a_{n}\right]$. Adding (9) and (7), which 
is

$$
\begin{aligned}
-\sum_{k=1}^{n}\left[\sum_{j=0}^{a_{k-1}-1} T\left[a_{0}, \ldots, a_{k-1}-j, j+a_{k}, \ldots, a_{n}\right]\right. & \\
& \left.+\sum_{j=0}^{a_{k}-1} T\left[a_{0}, \ldots, a_{k-1}+a_{k}-j, j, \ldots, a_{n}\right]\right]
\end{aligned}
$$

gives $-\sum_{k=1}^{n} \sum_{j=0}^{a_{k-1}+a_{k}-1} T\left[a_{0}, \ldots, a_{k-1}+a_{k}-j, j, \ldots, a_{n}\right]$ which cancels out (2) exactly.

Summing (8) gives $\left(a_{0}+a_{1}+\cdots+a_{n}\right) T\left[a_{0}, a_{1}, \ldots, a_{n}\right]$ and thus, because of the factor $\frac{1}{N}$ in $\sigma$, we get $T\left[a_{0}, a_{1}, \ldots, a_{n}\right]$.

To complete the proof, we need to show that (5), (12) and (13) add up to 0. This is where cyclicity of $T$ will be used.

As $T$ is cyclic, (12) can be rewritten as

$$
(-1)^{n+1} \sum_{j=0}^{a_{0}-1} T\left[a_{1}, \ldots, a_{n-1}, a_{n}+a_{0}-j, j\right] .
$$

Thus adding (13) and (12), which is

$$
\begin{aligned}
(-1)^{n+1} & {\left[\sum_{j=0}^{a_{n}-1} T\left[a_{1}, \ldots, a_{n-1}, a_{n}-j, a_{0}+j\right]\right.} \\
& \left.+\sum_{j=0}^{a_{0}-1} T\left[a_{1}, \ldots, a_{n-1}, a_{n}+a_{0}-j, j\right]\right],
\end{aligned}
$$

gives $(-1)^{n+1} \sum_{j=0}^{a_{n}+a_{0}-1} T\left[a_{1}, \ldots, a_{n-1}, a_{n}+a_{0}-j, j\right]$, which is the opposite of (5), and the result is proved.

\section{Other ORDERED DISCRETE SEMIGROUPS}

Now let $\mathcal{G}$ be a subgroup of $\mathbf{R}$ with the discrete topology, let $\mathcal{S}$ be the discrete semigroup $\mathcal{S}=\mathcal{G} \cap \mathbf{R}_{+}$and let $B=l^{1}(\mathcal{S})$. We will show that results identical to those for $l^{1}\left(\mathbf{Z}_{+}\right)$hold for $B$ by generalizing the proof of Proposition 5.3 .

We wish to define $\sigma: C^{n+1}\left(B, B^{\prime}\right) \rightarrow C^{n}\left(B, B^{\prime}\right)$.

In the degenerate case $a_{0}=a_{1}=\cdots=a_{n}=0$, let $\sigma T\left(z^{0}, \ldots, z^{0}\right)\left(z^{0}\right)=$ $T\left(z^{0}, \ldots, z^{0}\right)\left(z^{0}\right)$.

In the general case, let $v=\left(a_{0}, a_{1}, \ldots, a_{n}\right) \in \mathcal{S}^{n+1}$ be fixed (and not the degenerate vector $)$. For $T \in C^{n+1}\left(B, B^{\prime}\right)$, we define $\sigma T\left(z^{a_{0}}, \ldots, z^{a_{n-1}}\right)\left(z^{a_{n}}\right)$ in the following way.

We let $N=a_{0}+a_{1}+\cdots+a_{n}$, and we let $\mathcal{S}_{N}$ be the amenable group $\mathcal{G} /\langle N\rangle$, which we will identify with $\{s \in \mathcal{S}: 0 \leq s<N\}$ together with addition mod $N$. Let $\mu$ be an invariant mean on the amenable group $\mathcal{S}_{N}$.

For a vector $v=v(x) \in \mathcal{S}^{n+2}$, where $v(x)=\left(a_{0}, a_{1}, \ldots, a_{k}-x, x, \ldots, a_{n}\right)$ depends on $x$ (and even if $v=v(x)$ is constant), let $T[v]$ denote the expression 
$T\left(z^{a_{0}}, z^{a_{1}}, \ldots, z^{a_{k}-x}, z^{x}, \ldots, z^{a_{n-1}}\right)\left(z^{a_{n}}\right)$ and let $T[v(x)]_{a}^{b}$ denote the $l^{\infty}\left(\mathcal{S}_{N}\right)$ function defined by

$$
\begin{aligned}
T[v(x)]_{a}^{b}(s) & =T[v(s)] & & \text { if } a \leq s<b ; \\
& =0 & & \text { otherwise, }
\end{aligned}
$$

where it is assumed that $0 \leq a \leq N, 0 \leq b \leq N$.

Finally, for $y \in \mathcal{S}_{N}$, let $\chi_{y}$ be the characteristic function of $\left\{s \in \mathcal{S}_{N}: s<y\right\}$ in $l^{\infty}\left(\mathcal{S}_{N}\right)$, and let $\chi_{N}$ be the characteristic function of $\mathcal{S}_{N}\left(\right.$ also in $\left.l^{\infty}\left(\mathcal{S}_{N}\right)\right)$.

With the notation above, we define

$$
\sigma T\left(z^{a_{0}}, \ldots, z^{a_{n-1}}\right)\left(z^{a_{n}}\right)=\sum_{k=0}^{n}(-1)^{k+1} \mu\left(T\left[a_{0}, a_{1}, \ldots, a_{k}-x, x, \ldots, a_{n}\right]_{0}^{a_{k}}\right) .
$$

Note that $\mu\left(\chi_{a_{0}+a_{1}+\cdots+a_{n}}\right)=\mu\left(\chi_{N}\right)=1$.

Lemma 6.1. Let $T \in C C^{n+1}\left(B, B^{\prime}\right)$.

(1) If $n+1$ is odd, then $\sigma T$ is cyclic.

(2) If $n+1$ is even, then $\sigma T$ is cyclic if and only if $T$ is reduced.

Proof. This is virtually identical to the proof of Lemma 5.2.

Theorem 6.2. Let $T \in C C^{n}\left(B, B^{\prime}\right), n \geq 1$. Then $(\sigma \delta+\delta \sigma) T=T$.

Proof. The degenerate case is clear. For the general case, we follow the proof of Proposition 5.3. where all summations of the type

$$
\sum_{j=0}^{a_{k}-1} T\left[a_{0}, a_{1}, \ldots, a_{k}-x, x, \ldots, a_{n}\right]
$$

are replaced by $\mu\left(T\left[a_{0}, a_{1}, \ldots, a_{k}-x, x, \ldots, a_{n}\right]_{0}^{a_{k}}\right)$. We note that

$$
\begin{aligned}
& \mu\left(T\left[a_{0}, \ldots, a_{k-1}-x, x, a_{k}, \ldots, a_{n}\right]_{0}^{a_{k-1}}\right) \\
& +\mu\left(T\left[a_{0}, \ldots, a_{k-1}+a_{k}-x, x, \ldots, a_{n}\right]_{0}^{a_{k}}\right) \\
= & \mu\left(T\left[a_{0}, \ldots, a_{k-1}+a_{k}-x, x, \ldots, a_{n}\right]_{0}^{a_{k-1}+a_{k}}\right),
\end{aligned}
$$

because $\mu$ is translation invariant. Thus the cancellation of (2) with (7) and (9), which occurs in the proof of Proposition 5.3. still holds here. The other cancellations which occur in the proof of Proposition 5.3 still hold. Finally, because $\mu$ is translation invariant, it is clear from the definition of $\chi_{y}$ that $\sum_{k=0}^{n} \mu\left(\chi_{a_{k}}\right)=1$. Thus summing (8) now gives

$$
\begin{aligned}
\sum_{k=0}^{n} \mu\left(T\left[a_{0}, \ldots, a_{n}\right]_{0}^{a_{k}}\right) & =T\left[a_{0}, \ldots, a_{n}\right] \sum_{k=0}^{n} \mu\left(\chi_{a_{k}}\right) \\
& =T\left(z^{a_{0}}, \ldots, z^{a_{n-1}}\right)\left(z^{a_{n}}\right) .
\end{aligned}
$$

This completes the proof.

Thus we have

Theorem 6.3. Let $B=l^{1}(\mathcal{S})$, where $\mathcal{S}$ is defined as above.

(1) $\mathcal{H} C^{n}\left(B, B^{\prime}\right)=0$ when $n$ is odd, while it is one dimensional if $n$ is even $(n \geq 2)$. 
(2) The reduced cyclic cohomology of $B$ vanishes in all orders $n \geq 1$.

(3) Let $I$ be the maximal ideal of $B$ consisting of functions supported on the non-zero elements of $S$. Then $\mathcal{H} C^{n}\left(I, I^{\prime}\right)=0$ for all $n \geq 1$.

Proof. The first two statements follow directly from the previous theorem. To prove the third statement, we simply extend a cocycle $T \in C C^{n}(I)$ to $T \in C C^{n}(B)$ by first defining $T\left(z^{a_{0}}, \ldots, z^{a_{n-1}}\right)\left(z^{a_{n}}\right)=0$ if $a_{i}=0$ for some $i, 0 \leq i \leq n$, and then extending it by linearity. Applying $\sigma$ to this extended $T$ gives a cochain $S=\sigma T$. We can now restrict $S$ to $I$ (i.e. to $S \in C C^{n+1}(I)$ ), and the result follows from Theorem 6.2.

To deduce from Theorem 6.3 that $\mathcal{H}^{n}\left(B, B^{\prime}\right)=0$ for $n \geq 2$, we shall use part of Connes' exact sequence. We assume in what follows that $\mathcal{S} \cap(0, t)$ is infinite for every $t>0$, otherwise $l^{1}(\mathcal{S}) \cong l^{1}\left(\mathbf{Z}_{+}\right)$, a case which we have treated in Theorem 4.9 .

Lemma 6.4. Let I be the maximal ideal of B consisting of functions supported on the non-zero elements of $S$. Then $I^{\prime}$ is an injective right $I$-module.

Proof. By definition, an injective right $I$-module is an injective right $I^{\#}$-module [He0, Remark III.1.32], where $I^{\#}$ is the unitization of the Banach algebra $I$. Here $I^{\#}$ is isomorphic to $B$, and we will show that $I^{\prime}$ is an injective right $B$-module.

We let $I^{\prime}=l^{\infty}\left(\mathcal{S}_{0}\right)$ and $L\left(B, I^{\prime}\right)=l^{\infty}\left(\mathcal{S} \times \mathcal{S}_{0}\right)$, where $\mathcal{S}_{0}=\mathcal{S} \backslash\{0\}$.

To prove the result, we show that $I^{\prime}$ is a module direct summand of the (co-free) module $L\left(B, I^{\prime}\right)$ by giving an explicit module map $\lambda: L\left(B, I^{\prime}\right) \rightarrow I^{\prime}$ which is left inverse to the canonical inclusion of module $\mu^{*}: I^{\prime} \rightarrow L\left(B, I^{\prime}\right)$, where for $F \in I^{\prime}$ and $(s, t) \in\left(\mathcal{S} \times \mathcal{S}_{0}\right)$, we have $\mu^{*}(F)(s, t)=F(s+t)$. This will prove the result as $I^{\prime}$, being that a module direct summand of a co-free (hence injective) right $B$-module is itself a right injective $B$-module. See, for instance, [He0, Proposition III.1.31].

We begin by constructing a function $L: I^{\prime} \rightarrow \mathbf{C}$, which 'chooses' a value for $\lim _{s \rightarrow 0} F(s)$, where $F \in I^{\prime}$. To this end, consider an ultrafilter $\mathcal{U}$ which contains the sets $\{S \cap(0, t): t>0\}$, and let $L(F)=\lim _{\mathcal{U}} F(s)$ be the limit of $F(s)$ along the ultrafilter $\mathcal{U}$.

The map $\lambda: L\left(B, I^{\prime}\right) \rightarrow I^{\prime}$ is defined by $\lambda(T)(s)=L(x \mapsto T(s-x, x))$, where $T \in L\left(B, I^{\prime}\right)$ and $s \in \mathcal{S}_{0}$. Note that here $x \mapsto T(s-x, x)$ is the element of $I^{\prime}=l^{\infty}\left(\mathcal{S}_{0}\right)$ taking value $T(s-x, x)$ at $x \leq s$, and taking value 0 at each $x>s$.

The map $\lambda$ is a right inverse to $\mu^{*}$ as we have, for $s \in \mathcal{S}_{0}$,

$$
\begin{aligned}
\lambda\left(\mu^{*}(F)\right)(s) \quad & L\left(x \mapsto \mu^{*}(F)((s-x), x)\right) \\
& =L(x \mapsto F(s-x+x)) \\
& =L(x \mapsto F(s))=F(s) .
\end{aligned}
$$

We next need to show that $\lambda$ is a module map. It is straigthforward to verify that $\lambda$ is bounded and linear. To show that it commutes with the modules action by $B$, it therefore suffices to show that it commutes with the action of the point masses $\left\{\delta_{t}: t \in S\right\}$. We have

$$
\lambda\left(T * \delta_{t}\right)(s)=L\left(x \mapsto\left(T * \delta_{t}\right)(s-x, x)\right)=L(x \mapsto T(t+s-x, x)),
$$

where the last equality holds because of the definition of $L$. It is readily seen that $L(x \mapsto T(t+s-x, x))=\lambda(T)(t+s)=\left(\lambda(T) * \delta_{t}\right)(s)$ which completes the proof. 
Corollary 6.5. The portion of Connes' exact sequence starting with $\mathcal{H} C^{2}(I)$ is exact.

Proof. In a way similar to [He1, Example 21], we note that $I^{\prime}$ is injective and hence $\operatorname{Ext}_{I}^{n}\left(\mathbf{C}, I^{\prime}\right)=0$ for all $n>0[\mathrm{He} 0$, Proposition 3.4.5]. Thus the result follows from [ [He1, Theorem 11] or, more precisely, from the brief discussion following this theorem.

Theorem 6.6. $\mathcal{H}^{n}\left(B, B^{\prime}\right)=0$ for $n \geq 2$.

Proof. The proof is identical to that of Theorem 4.9. We show that $\mathcal{H}^{n}\left(I, I^{\prime}\right)$ vanishes for $n \geq 2$ using the portion of Connes' exact sequence which exists by Corollary 6.5, and the vanishing of $\mathcal{H} C^{n}(I)$ (Theorem 6.3). Now the proof of GW, Proposition 2.1], which was done for $l^{1}\left(\mathbf{Z}_{+}\right)$, also holds for $B$ (the crucial point being that $\operatorname{Ext}_{I}^{n}\left(\mathbf{C}, I^{\prime}\right)=0$, a fact we already used in the proof of the previous corollary). Therefore we have $\mathcal{H}^{n}\left(B, B^{\prime}\right)=\mathcal{H}^{n}\left(I, I^{\prime}\right)$. This completes the proof.

\section{Conclusion}

In the generalization to $l^{1}(\mathcal{S})$, we have used a translation invariant mean on part of the semigroup (considered as a group $\mathcal{S}_{N}$ ), together with the fact that the natural ordering of $\mathcal{S}$ is such that we have

$$
\mu\left(\chi_{a}\right)+\mu\left(\chi_{b}\right)=\mu\left(\chi_{a+b}\right) .
$$

Such a property does not always hold. For instance, it is not possible to extend this method to $\mathbf{Z}_{+}^{k}$ for $k \geq 2$ which is in agreement with the fact, shown in [GW], that the second simplicial cohomology group of $l^{1}\left(\mathbf{Z}_{+}^{2}\right)$ does not vanish.

It is natural to ask if the proofs can be adapted to the case of $L^{1}\left(\mathbf{R}_{+}\right)$and whether or not similar results are true in the case of the disc algebra. It seems

likely that the answer is yes in the first case while we expect a different answer for the disc algebra. However the work on these questions is not yet completed.

\section{ACKNOWLEDGMENT}

The first author thanks the University of Newcastle upon Tyne for its kind hospitality while this paper was being written, and aknowledges the support of grant 184058-00 of Canada's NSERC.

\section{REFERENCES}

[BCD] W.G. Bade, P.C. Jr Curtis, and H.G. Dales, Amenability and weak amenability for Beurling and Lipschitz algebras, Proc. London Math. Soc. (3), 55 (1987), 359-377. MR0896225 (88f:46098)

[BD] F.F. Bonsall and J. Duncan, Complete normed algebras, Springer-Verlag, New York, 1973. MR0423029 (54:11013)

[DaDu] H. G. Dales and J. Duncan, Second order cohomology in groups of some semigroup algebras, Banach Algebras '97, Proc. 13th. Internat. Confer. on Banach Algebras, 101-117, Walter de Gruyter, Berlin, 1998. MR.1656601 (99m:46168)

[GW] F. Gourdeau and M. C. White, Vanishing of the third simplicial cohomology group of $l^{1}\left(\mathbf{Z}_{+}\right)$, Trans. AMS (353), 5 (2001), 2003-2017. MR1813605 (2001m:46163)

[He0] A. Ya. Helemskii, The homology of Banach and topological algebras. Kluwer Academic Publishers, Dordrecht, 1989. MR1093462 (92d:46178)

[He1] A. Ya. Helemskii, Banach cyclic (co)homology and the Connes-Tzygan exact sequence, J. London Math. Soc., (2) 46, (1992), 449-462. MR1190429 (94a:46105) 
[BEJ1] B.E. Johnson, Cohomology in Banach algebras, Mem. Amer. Math. Soc., 127 (1972). MR0374934(51:11130)

[BEJ2] B.E. Johnson, Higher-dimensional weak amenability, Studia Math., 123 (1997), 2, 117134. MR 1439025 (98f:46046)

[No] D.G. Northcott, Introduction to homological algebra, Cambridge University Press, 1962. MR0118752(22:9523)

Département de Mathématiques et de Statistique, Université laval, Cité UniversiTAIRE, QuÉBec, CANAda G1K 7P4

E-mail address: Frederic.Gourdeau@mat.ulaval.ca

Department of Mathematics, University of Newcastle, Newcastle upon Tyne, Ne1 7RU, ENGLAND

Department of Mathematics, University of Newcastle, Newcastle upon Tyne, Ne1 7RU, ENGLAND

E-mail address: Michael.White@ncl.ac.uk 